\title{
Uncertainty and economic growth in a stochastic R\&D model
}

\author{
Fuke $\mathrm{Wu}^{1 *}$ Xuerong $\mathrm{Mao}^{2 \dagger}$ Juliang $\mathrm{Yin}^{3}$ \\ ${ }^{1}$ Department of Mathematics \\ Huazhong University of Science and Technology, Wuhan, Hubei 430074, P.R.China \\ ${ }^{2}$ Department of Statistics and Modelling Science, University of Strathclyde, Glasgow G1 1XH, UK \\ ${ }^{3}$ Department of Statistics, Jinan University, Guangzhou, Guangdong 510630, P.R.China
}

\begin{abstract}
The paper examines an R\&D model with uncertainty from the population growth, which is a stochastic cooperative Lotka-Volterra system, and obtains a sufficiently condition for the existence of the globally positive solution. The long-run growth rate of the economic system is ultimately bounded in mean and fluctuation of its growth will not be faster than the polynomial growth. When uncertainty of the population growth, in comparison with its expectation, is sufficiently large, the growth rate of the technological progress and the capital accumulation will converge to zero. Inversely, when uncertainty of the population growth is sufficiently small or its expected growth rate is sufficiently high, the economic growth rate will not decay faster than the polynomial speed. The paper explicitly computes the sample average of the growth rates of both the technology and the capital accumulation in time and compares them with their counterparts in the corresponding deterministic model. JEL classification: C62; O41;

Keywords: Lotka-Volterra system; Brownian motion; R\&D model; Polynomial growth; Stochastic differential equation; Itô formula
\end{abstract}

\footnotetext{
${ }^{*}$ Corresponding author. E-mail: wufuke@mail.hust.edu.cn

${ }^{\dagger} E$-mial: xuerong@stams.strath.ac.uk
} 


\section{Introduction}

The central purpose of the economic growth theories is to understand the factors behind long-run growth and to explain differences in growth performances of economies. After the seminal papers worked by Bourguignon [4] and Merton [15] introduce uncertainty into the neoclassical model of growth developed by Solow [20], the increasing papers realize the effect of uncertainty on the growth performances of economy. For example, Binder and Pesaran [3] show the expectation of the steady-state distribution of the capital-output ratio under the stochastic model exceeds the value of steady-state capital-output ratio under the deterministic model. Canton [6] constructs a stochastic two-sector model of endogenous growth to analyze impact of uncertainty on long-run economic growth, and shows that economic growth is higher since people devote more time to learning activities in an uncertain economic environment. In these papers, uncertainty plays a positive role during the economic growth process. However, in Hek's

[9] model, uncertainty plays a negative role. He examines uncertainty from creation of knowledge and obtains the negative relation between uncertainty and growth.

Some empirical work also shows confusing results between growth and uncertainty. For example, considering a sample of 47 countries with data covering the 1950-1977 period, Kormendi and Meguire [11] show that more volatile countries - as measured by the standard deviation of their growth rates - grow at a higher rate. Grier and Tullock [7] use panel data techniques on a sample of 113 countries covering a period from 1951-1980 and obtain the same results as these of Kormendi and Meguire. While, Zarnowitz and Moore [21] find that the standard deviation of GNP tends to be higher during periods of lower growth. Ramsey and Ramsey [17] find a sample of 92 countries as well as in a sample of OECD countries that countries with higher uncertainty have lower growth.

The paper introduces uncertainty from the population growth into the R\&D model developed by D. Romer [18] and examines its effect on economic growth. It is wellknown that the population growth is always an important factor in neoclassical economic growth models. In Solow [20] and Ramsey [16] models, the long-run growth rate of the aggregate capital accumulation completely depends on the exogenous technological progress and population growth rate. To study endogenous economic growth, many models (for example, [18], P96 and [1], [8] and [10]) see technological progress as a production process like production of output. In these models, the long-run economic growth rate is completely determined by the population growth rate. Applying 
Itô formula, the paper incorporates uncertainty of population growth into a simplified version of these models.

Under a condition under which there exists a uniquely globally steady state in the deterministic R\&D model, where the long-run growth rates of both the technological progress and capital accumulation are positive constants, the corresponding stochastic technology and capital accumulation still show positive growth. Their growth rates will not grow faster than the polynomial speed. When uncertainty is sufficiently large or the expected population growth rate is sufficiently low, the long-run growth rates of both the technological progress and capital accumulation will converge to zero, which means the economy will stop growing and be in a stagnant state. Inversely, when uncertainty is sufficiently small or the expected population growth rate is sufficiently high, the growth of the economic system will not decay faster than polynomial speed and the long-run expected growth rates of both the technological progress and the capital accumulation are controlled by the difference between the expected population growth rate and its uncertainty. The less difference means the less expected growth rates.

The most important contribution of this paper is that we explicitly compute the sample average of the growth rates of both the technological progress and the capital accumulation in time, which may be expressed as the difference between the steadystate values in the corresponding deterministic model and a product of a positive number and the variance of the uncertainty, so it is very easy to see that the uncertainty decreases the sample average of the economic growth rate. If the variance of the uncertainty is zero, the sample average of the growth rates of both the technological progress and the capital accumulation equal the steady-state values in the deterministic model.

In the paper, we use the recent mathematical technique on stochastic Lotka-Volterra system to prove the existence and uniqueness of the globally positive solution and estimate its asymptotic properties. Some theoretical results may be found in [2] and [14]. The paper applies these results into the stochastic R\&D model and obtains some more precious estimation and asymptotic properties.

To compare the stochastic R\&D model with its deterministic version, in Section two, the paper reviews the simplified deterministic R\&D model developed by Romer [18] and its main results. In Section three, we introduce uncertainty into the deterministic model and prove the existence and uniqueness of the globally positive solution and estimate the properties of the solution. According to the asymptotic properties, we 
explicitly compute the sample average of the growth rates of both the technological progress and the capital accumulation in time and compare them with the deterministic counterparts. The last section is concluding remark.

\section{Retrospect of a deterministic R\&D model}

Firstly, we shortly review a simplified version of the deterministic models of $R \& D$ and growth developed by P. Romer [19], Grossman and Helpman [8], Aghion and Howitt [1] and Jones [10], which is wholly described by D. Romer ([18], P96). The models involves four variables: labor $(\mathrm{L})$, capital $(\mathrm{K})$, technology $(\mathrm{A})$ and output $(\mathrm{Y})$. There are two sectors, a goods-producing sector where output is produced and an R\&D sector where additions to the stock of knowledge (technological progress) are made. Fraction $a_{L}$ of the labor force is used in the $\mathrm{R} \& \mathrm{D}$ sector and fraction $1-a_{L}$ in the goods-producing sector; Similarly, fraction $a_{K}$ of the capital stock is used in R\&D and the rest in goods production. Both sectors use the full stock of knowledge, $A$.

In the two sectors, the production functions are, respectively

$$
\begin{aligned}
& Y(t)=\left[\left(1-a_{K}\right) K(t)\right]^{\alpha}\left[A(t)\left(1-a_{L}\right) L(t)\right]^{1-\alpha}=C_{Y} K^{\alpha}(t)(A(t) L(t))^{\alpha^{\prime}}, \\
& \dot{A}(t)=B\left[a_{K} K(t)\right]^{\xi}\left[a_{L} L(t)\right]^{\eta} A^{1+\theta}(t)=C_{K} K^{\xi}(t) A^{1+\theta}(t) L^{\eta}(t),
\end{aligned}
$$

where $C_{Y}=\left(1-a_{K}\right)^{\alpha}\left(1-a_{L}\right)^{1-\alpha}, C_{K}=B a_{K}^{\xi} a_{L}^{\eta}, \alpha^{\prime}=1-\alpha, \alpha \in(0,1)$ and $\xi, \eta>0$, $B>0$ is a parameter which measures efficiency in R\&D sector. Population growth is exogenous and $\dot{L}(t)=n L(t)$.

Set $x(t)=\dot{A}(t) / A(t), y(t)=\dot{K}(t) / K(t)=s Y(t) / K(t)$, i.e., they respectively represent the growth rates of technological progress and capital accumulation, where $s \in(0,1)$ is the saving rate and the depreciation rate of capital $K(t)$ is not considered. Then, according to the productions functions and definition of $x(t)$ and $y(t)$, the following cooperative Lotka-Volterra system may represent this economic system.

$$
(\mathrm{CP}) \quad\left\{\begin{array}{l}
\dot{x}(t)=x(t)[\theta x(t)+\xi y(t)+n \eta] \\
\dot{y}(t)=\alpha^{\prime} y(t)[x(t)-y(t)+n],
\end{array}\right.
$$

which has following properties:

(i) If $\theta+\xi<0$, the system exists a uniquely steady state $\left(x^{*}, y^{*}\right)$, where

$$
x^{*}=-\frac{n(\eta+\xi)}{\theta+\xi}, \quad y^{*}=-\frac{n(\eta-\theta)}{\theta+\xi} .
$$


Furthermore, the system exhibits global stability.

(ii) if $\theta<0$ and $\theta+\xi>0$, or $\theta+\xi=0<n$, the system is constant divergent. Regardless of where the economy starts, it eventually enters the region between the line $\theta x+\xi y+n \eta=0$ and $x-y+n=0$, and hence the growth increases continually. Further, when $\theta>0$, the economy will explode in the finite time.

(iii) if $\theta+\xi=0$ and $n=0$, the system will converge to one of points on the line $y=x$.

The properties imply the technological progress and the capital accumulation will show exponential growth at the rates $x^{*}$ and $y^{*}$ when $\theta+\xi<0$. When $\theta<0$ and $\theta+\xi>0$, or $\theta+\xi=0<n$, both the technology and the capital accumulation will accelerate. If $\theta>0$, that is, technology has more contribution for additions of the new knowledge, the economy will explode in finite time. However, in real economy, we do not see the explosion. Maybe some other factors suppress the explosion. In our next paper, we will show how uncertainty to suppress the explosion. When $\theta+\xi=0$ and $n=0$, these growth rates are constants, which cannot be determined.

The main objective of the paper is to examine whether the result will be changed under the condition $\theta+\xi<0$ if uncertainty is introduced into the model. If the result will be changed, we hope to analyze the properties of the changed result and compare them with their deterministic counterparts. Therefore, we begin to consider the stochastic R\&D model.

\section{The uncertainty model}

Now we incorporate uncertainty into the above R\&D model. Throughout this paper, let $(\Omega, \mathcal{F}, P)$ be a complete probability space with a filtration $\left\{\mathcal{F}_{t}\right\}_{t>0}$ satisfying the usual conditions, that is to say, it is right continuous and increasing while $\mathcal{F}_{0}$ contains all P-null sets. Let $w(t)$ denote a scalar Brownian motion defined on this probability space. The paper also defines $\mathbf{R}_{+}^{2}=\left\{(x, y) \in \mathbf{R}^{2}: x>0, y>0\right\}$.

In the stochastic R\&D model, the uncertainty comes from the population growth, which satisfies the following geometric Brownian motion:

$$
d L(t)=L(t)(n d t+\sigma d w(t))
$$

where $n$ is defined as before and represents the expected population growth rate. 
Using Itô formula,

$$
\begin{aligned}
\frac{d x(t)}{x(t)} & =\xi \frac{d K(t)}{K(t)}+\theta \frac{d A(t)}{A(t)}+\eta \frac{d L(t)}{L(t)}-\frac{\eta \eta^{\prime}}{2} \frac{(d L(t))^{2}}{L^{2}(t)} \\
& =\left(\theta x(t)+\xi y(t)+b_{x}\right) d t+\eta \sigma d w(t), \\
\frac{d y(t)}{y(t)} & =\left(\alpha^{\prime} x(t)-\alpha^{\prime} y(t)+b_{y}\right) d t+\alpha^{\prime} \sigma d w(t),
\end{aligned}
$$

where $b_{x}=\eta n-\frac{\eta \eta^{\prime}}{2} \sigma^{2}, b_{y}=\alpha^{\prime} n-\frac{\alpha^{\prime} \alpha}{2} \sigma^{2}$, and $\eta^{\prime}=1-\eta$.

Define

$$
X(t)=(x(t), y(t))^{T}, A=\left[\begin{array}{cc}
\theta & \xi \\
\alpha^{\prime} & -\alpha^{\prime}
\end{array}\right], b=\left(b_{x}, b_{y}\right)^{T}, \beta=\left(\eta \sigma, \alpha^{\prime} \sigma\right)^{T},
$$

then the equation on $(x(t), y(t))$ may be written as:

$$
(\mathrm{SP}) \quad d X(t)=\operatorname{diag}(x(t), y(t))[(A X(t)+b) d t+\beta d w(t)]
$$

which is a stochastic cooperative Lotka-Volterra system.

The stochastic Lotka-Volterra system with some different extension has recently received a lot of attention because it may simulate many biological and other processes. Bahar and Mao [2] use this system with time delay to model population growth and find the sufficiently condition for existence of the globally positive solution and show that the solution is ultimately bounded in mean. When uncertainty is sufficiently large, they find the system will converge to trivial solution, which means the population, as a group, will become extinct. Pang, et al [14] discuss asymptotic properties of the system without time delay and find that the system will at most grow or decay with the polynomial speed, that is to say, the system will not violently fluctuate if uncertainty is constrained.

In the paper, we apply their results to the system (SP) and hope to obtain some more precious results. Firstly, we hope to know whether the condition $\theta+\xi<0$ may still ensure the solution of Eq. (3) is positive since its deterministic counterpart is positive, which means that the technology and the capital accumulation shows the positive growth. With respect to this, the paper has the following theorem.

Theorem 1 when $\theta+\xi<0$, for any given initial value $x(0), y(0)>0$, there is a uniquely global solution $(x(t), y(t))$ to the system $(S P)$ on $t \geq 0$ and the solution will remain in $\mathbf{R}_{+}^{2}$ with probability 1 , namely $x(t), y(t)>0$ for all $t \geq 0$ almost surely. 
Proof According to Pang, et al ([14] Theorem 2.1) and Bahar and Mao ([2] Corollary 2.3), if there exist positive constants $c_{1}, c_{2}$ such that $-\left(\bar{C} A+A^{T} \bar{C}\right)$ is positive definite, where $\bar{C}=\operatorname{diag}\left(c_{1}, c_{2}\right)$ is a diagonal matrix, then there exists a uniquely globally positive solution $(x(t), y(t))^{T}$ to the system (SP) on $t \geq 0$.

Because $\theta+\xi<0, \xi, \alpha^{\prime}>0, A^{\prime}$ 's inverse matrix

$$
A^{-1}=\frac{1}{\alpha^{\prime}(\theta+\xi)}\left[\begin{array}{cc}
\alpha^{\prime} & \xi \\
\alpha^{\prime} & -\theta
\end{array}\right]<0,
$$

i.e., $-A$ is a nonsingular M-matrix. According to the nonsingular M-matrix's properties (see [5], Chapter 6), there exists a positive diagonal matrix $\bar{C}$ such that $-\left(\bar{C} A+A^{T} \bar{C}\right)$ is positive definite. The desired result may be obtained.

In the corresponding deterministic model, the condition $\theta+\xi<0$ may ensure the system exist a positive equilibrium position and the equilibrium position is a globally steady state. In the stochastic model, because the equilibrium position (see [12] (P110) for the definition of the equilibrium position or the trivial solution) requires both the drift and diffusion coefficients are zero at this point, (SP) does not exist non-zero equilibrium position. Therefore, it is impossible for (SP) to exist a positive steady state, but the condition can still ensure the technology and capital accumulation show positive growth. Although the explicit long-run growth rates can not be computed as that in the deterministic model, we may still make some estimation for them. The following theorem shows the solution is ultimately bounded in mean in the long run.

Theorem 2 when $\theta+\xi<0$, the expected long-run growth rates of both the technology and the capital accumulation are ultimate bounded

Proof By the theorem 1, the solution $(x(t), y(t)) \in \mathbf{R}_{+}^{2}$ a.s. Define

$$
V(x(t), y(t))=x(t)-\frac{2 \theta+\xi}{\alpha^{\prime}} y(t) .
$$

By the Itô formula, we have

$$
\begin{aligned}
d V(x(t), y(t))= & {\left[\theta x^{2}(t)-2 \theta x(t) y(t)+(2 \theta+\xi) y^{2}(t)+b_{x} x(t)-\frac{2 \theta+\xi}{\alpha^{\prime}} b_{y} y(t)\right] d t } \\
& +\sigma[\eta x(t)-(2 \theta+\xi) y(t)] d w(t) .
\end{aligned}
$$

For any $\gamma>0$, applying the Itô formula again,

$$
\begin{aligned}
d\left[e^{\gamma t} V(x(t), y(t))\right] & =e^{\gamma t}[r V(x(t), y(t))+d V(x(t), y(t))] \\
& =e^{\gamma t} L(x(t), y(t)) d t+\sigma e^{\gamma t}[\eta x(t)-(2 \theta+\xi) y(t)] d w(t),
\end{aligned}
$$


where

$$
L(x, y)=\left[\theta x^{2}-2 \theta x y+(2 \theta+\xi) y^{2}+\left(\gamma+n \eta-\frac{\eta \eta^{\prime}}{2} \sigma^{2}\right) x-(2 \theta+\xi)\left(\frac{\gamma}{\alpha^{\prime}}+n-\frac{\alpha}{2} \sigma^{2}\right) y\right],
$$

which is a concave function, so there exists a maximal value $L_{\max }$ for the function. Therefore,

This yields

$$
e^{\gamma t} E V(x(t), y(t)) \leq V(x(0), y(0))+\frac{L_{\max }}{\gamma}\left(e^{\gamma t}-1\right)
$$

$$
\limsup _{t \rightarrow+\infty} E V(x(t), y(t)) \leq \frac{L_{\max }}{\gamma}
$$

The result means

$$
\max \{E x, E y\} \leq \frac{L_{\max }}{\gamma\left(1-\frac{2 \theta+\xi}{\alpha^{\prime}}\right)},
$$

that is to say, the expected growth rates of both the technology and the capital accumulation are bounded in the long run. This completes the proof.

Now we precisely discuss the upper bound. It is a key to determine $L_{\max } / \gamma$. Firstly, if $\max \left\{b_{x}, b_{y}\right\}<0$, which is equivalent to $2 n<\min \left\{\alpha, \eta^{\prime}\right\} \sigma^{2}$, then choosing $\gamma=$ $-\max \left\{b_{x}, b_{y}\right\}$ yields $L_{\max }=0$, so $\lim \sup _{t \rightarrow \infty} E V(x(t), y(t)) \leq 0$. Noting $x(t), y(t)>0$ when $\theta+\xi<0$, so $\lim \sup _{t \rightarrow \infty} E V(x(t), y(t))=0$. Therefore, $\lim _{t \rightarrow \infty} V(x(t), y(t))=0$, which means that $\lim _{t \rightarrow \infty} x(t)=\lim _{t \rightarrow \infty} y(t)=0$. Therefore, the paper may obtain the following corollary.

Corrollary 1 when $\theta+\xi<0$ is retained, if $2 n<\min \left\{\alpha, \eta^{\prime}\right\} \sigma^{2}$, then

$$
\lim _{t \rightarrow \infty} x(t)=\lim _{t \rightarrow \infty} y(t)=0, \text { a.s. }
$$

This result shows the technology and capital accumulation will stop growing if the uncertainty is relatively large or the expected population growth rate is relatively low. We will continually discuss this result in Theorem 3.

Then we examines the upper bound when $\min \left\{b_{x}, b_{y}\right\}>0$ that means $2 n>\max \left\{\alpha, \eta^{\prime}\right\} \sigma^{2}$, that is to say, the uncertainty is relatively small or the expected population growth rate is relatively low. Applying Lagrange maximal principle, when

$$
\tilde{x}=\tilde{y}-\frac{\gamma+b_{x}}{2 \theta}, \quad \tilde{y}=-\frac{\gamma+b_{x}}{2(\theta+\xi)}+\frac{(2 \theta+\xi)\left(\gamma+b_{y}\right)}{2 \alpha^{\prime}(\theta+\xi)},
$$

the function $L(x, y)$ reaches its maximal value. $\min \left\{b_{x}, b_{y}\right\}>0$ means that $\tilde{x}, \tilde{y}>0$, so

$$
\begin{aligned}
L_{\max }= & -\frac{1}{4(\theta+\xi)}\left[\gamma+b_{x}-\frac{2 \theta+\xi}{\alpha^{\prime}}\left(\gamma+b_{y}\right)\right]^{2}-\frac{1}{4 \theta}\left(\gamma+b_{x}\right)^{2} \\
& \leq-\frac{1}{4}\left[\frac{1}{\theta+\xi}\left(\frac{\alpha^{\prime}-(2 \theta+\xi)}{\alpha^{\prime}}\right)^{2}+\frac{1}{\theta}\right] \max \left\{\left(\gamma+b_{x}\right)^{2},\left(\gamma+b_{y}\right)^{2}\right\} .
\end{aligned}
$$


Choose $\gamma=\max \left\{b_{x}, b_{y}\right\}$, then

$$
\frac{L_{\max }}{\gamma} \leq-\left[\frac{1}{\theta+\xi}\left(\frac{\alpha^{\prime}-(2 \theta+\xi)}{\alpha^{\prime}}\right)^{2}+\frac{1}{\theta}\right] \max \left\{b_{x}, b_{y}\right\}
$$

Substituting this into the result of Theorem 2, we may deduce the upper bound of the long-run growth rates of both the technological progress and the capital accumulation depends on the difference between the expected population growth rate and its uncertainty, which may express as

Corrollary 2 when $\theta+\xi<0$ is retained, if $2 n>\max \left\{\alpha, \eta^{\prime}\right\} \sigma^{2}$, then

$\limsup _{t \rightarrow \infty} E x(t) \leq \frac{-\alpha^{\prime}}{\alpha^{\prime}-(2 \theta+\xi)}\left[\frac{1}{\theta+\xi}\left(\frac{\alpha^{\prime}-(2 \theta+\xi)}{\alpha^{\prime}}\right)^{2}+\frac{1}{\theta}\right] \max \left\{\eta n-\frac{\eta \eta^{\prime}}{2} \sigma^{2}, \alpha^{\prime} n-\frac{\alpha \alpha^{\prime}}{2} \sigma^{2}\right\}$, $\limsup _{t \rightarrow \infty} E y(t) \leq \frac{-\alpha^{\prime}}{\alpha^{\prime}-(2 \theta+\xi)}\left[\frac{1}{\theta+\xi}\left(\frac{\alpha^{\prime}-(2 \theta+\xi)}{\alpha^{\prime}}\right)^{2}+\frac{1}{\theta}\right] \max \left\{\eta n-\frac{\eta \eta^{\prime}}{2} \sigma^{2}, \alpha^{\prime} n-\frac{\alpha \alpha^{\prime}}{2} \sigma^{2}\right\}$.

Therefore, the difference between the expected population growth rate and its uncertainty may control the expected growth rates of both the technological progress and the capital accumulation. the less $2 n-\max \left\{\alpha, \eta^{\prime}\right\} \sigma^{2}$ means the less the expected economic growth rate.

Because we only obtain an upper bound, from this result we can not decide whether the higher expected population growth rate or the smaller uncertainty can increase the expected growth rates of both the technological progress and capital accumulation. Later we will find the higher expected growth rate or the smaller uncertainty will increase the sample average of the growth rates of both the technological progress and capital accumulation.

According to the expression of (SP), the origin point is its only equilibrium position. If the trivial solution is stable, the system will converge to the equilibrium position, which shows that the growth rates of the technology and capital accumulation will converge to zero and the economy will stop growing and be in stagnant state. With respect to the equilibrium position, we have the following result.

Theorem 3 When $\theta+\xi<0$ is retained, if $2 n<\left[1-\left(\sqrt{\alpha^{\prime}}-\sqrt{\eta}\right)^{2}\right] \sigma^{2}$, then

$$
\lim _{t \rightarrow+\infty} x(t)=0, \quad \lim _{t \rightarrow+\infty} y(t)=0, \text { a.s. }
$$

Proof We still define the same function

$$
V(x(t), y(t))=x(t)-\frac{2 \theta+\xi}{\alpha^{\prime}} y(t),
$$


by the Itô formula for $\log V(x(t), y(t))$, then we obtain

$$
\begin{aligned}
& d \log V(x(t), y(t))=\frac{d V(x(t), y(t))}{V(x(t), y(t))}-\frac{[d V(x(t), y(t))]^{2}}{2 V^{2}(x(t), y(t))} \\
\leq & \frac{1}{V(x(t), y(t))}\left[\left(b_{x} x(t)-\frac{2 \theta+\xi}{\alpha^{\prime}} b_{y} y(t)\right) d t+\sigma(\eta x(t)-(2 \theta+\xi) y(t)) d w(t)\right] \\
& -\frac{1}{2 V^{2}(x(t), y(t))}\left[\eta^{2} \sigma^{2} x^{2}(t)+\sigma^{2}(2 \theta+\xi)^{2} y^{2}(t)-2 \eta \sigma(2 \theta+\xi) x(t) y(t)\right] d t \\
= & \frac{1}{2 V^{2}(x(t), y(t))} I(x(t), y(t)) d t+\frac{\sigma}{V(x(t), y(t))}[\eta x(t)-(2 \theta+\xi) y(t)] d w(t),
\end{aligned}
$$

where

$I(x, y)=\eta\left(2 n-\sigma^{2}\right) x^{2}-\frac{(2 \theta+\xi)\left(\alpha^{\prime}+\eta\right)}{\alpha^{\prime}}\left[2 n-\left(1-\frac{\left(\alpha^{\prime}-\eta\right)^{2}}{\alpha^{\prime}+\eta}\right) \sigma^{2}\right] x y+\frac{(2 \theta+\xi)^{2}}{\alpha^{\prime}}\left(2 n-\sigma^{2}\right) y^{2}$.

If there exists a constant $\varphi>0$ such as

$$
I(x(t), y(t)) \leq-\varphi V^{2}(x(t), y(t))
$$

then

$$
d \log V(x(t), y(t)) \leq-\frac{\varphi}{2} d t+M(t)
$$

where $M(t)$ is a martingale defined

$$
M(t)=\frac{\sigma}{V(x(t), y(t))}[\eta x(t)-(2 \theta+\xi) y(t)] d w(t) .
$$

The quadratic variation of this martingale is

$$
\begin{aligned}
\langle M(t), M(t)\rangle_{t} & =\int_{0}^{t} \frac{\sigma^{2}}{V^{2}(x(t), y(t))}[\eta x(t)-(2 \theta+\xi) y(t)]^{2} d w(t) d s \\
& \leq \max \left\{\alpha^{\prime 2}, \eta^{2}\right\} \sigma^{2} t
\end{aligned}
$$

Hence

$$
\limsup _{t \rightarrow+\infty} \frac{\langle M(t), M(t)\rangle_{t}}{t} \leq \max \left\{\alpha^{\prime 2}, \eta^{2}\right\} \sigma^{2} \quad \text { a.s. }
$$

By the strong law of large number for martingale (see [12] P12),

$$
\limsup _{t \rightarrow+\infty} \frac{1}{t} \log V(x(t), y(t)) \leq-\frac{\varphi}{2} \quad \text { a.s. }
$$

Therefore,

$$
\limsup _{t \rightarrow+\infty} V(x(t), y(t))=0 \quad \text { a.s. }
$$


which means

$$
\lim _{t \rightarrow+\infty} x(t)=0, \quad \lim _{t \rightarrow+\infty} y(t)=0 \text {,a.s. }
$$

since $x(t)>0, y(t)>0$. Therefore, it is a key to find $\varphi$. We consider it by two steps. Firstly, when

$$
2 n-\sigma^{2}<-\frac{\left(\alpha^{\prime}-\eta\right)^{2}}{\alpha^{\prime}+\eta} \sigma^{2}
$$

choose

$$
\varphi=\min \left\{\eta, \alpha^{\prime}, \frac{\alpha^{\prime}+\eta}{2}-\frac{\left(\alpha^{\prime}-\eta\right)^{2} \sigma^{2}}{2\left(\sigma^{2}-2 n\right)}\right\}\left(\sigma^{2}-2 n\right),
$$

then (4) will be satisfied.

Secondly, define $-\lambda=\left(2 n-\sigma^{2}\right)^{2}+2\left(2 n-\sigma^{2}\right)\left(\alpha^{\prime}+\eta\right) \sigma^{2}+\left(\alpha^{\prime}-\eta\right)^{2} \sigma^{4}$, When $-\left(\alpha^{\prime}-\eta\right)^{2} /\left(\alpha^{\prime}+\eta\right)<2 n-\sigma^{2}<\left[1-\left(\sqrt{\alpha^{\prime}}-\sqrt{\eta}\right)^{2}\right] \sigma^{2}, \lambda>0$, then choose

$$
\varphi=\min \left\{\eta\left(\sigma^{2}-2 n\right), \alpha^{\prime}\left(\sigma^{2}-2 n\right), \frac{\lambda\left(\alpha^{\prime}+\eta\right)}{4 \sigma^{2}}\right\},
$$

then (4) will be satisfied. Therefore, the desired assertion is obtained.

Comparing this result with Corollary 1, the result may be rewritten as

Corrollary 3 When $\theta+\xi<0$ is retained, if $2 n<\left(\left[1-\left(\sqrt{\alpha^{\prime}}-\sqrt{\eta}\right)^{2}\right] \vee \min \left\{\alpha^{\prime}, \eta\right\}\right) \sigma^{2}$, then

$$
\lim _{t \rightarrow+\infty} x(t)=0, \quad \lim _{t \rightarrow+\infty} y(t)=0, \text { a.s. }
$$

Although in the corresponding deterministic model as in the section two of this paper, the steady-state growth rates of both technology and capital accumulation are completely determined by the population growth rate when $\theta+\xi<0$. If there is positive population growth rate, the economy will show positive growth rate. The higher population growth rate means the higher economic growth rate. In a stochastic environment, because of effect of uncertainty, if the expected population growth rate satisfies $2 n<\left(\left[1-\left(\sqrt{\alpha^{\prime}}-\sqrt{\eta}\right)^{2}\right] \vee \min \left\{\alpha^{\prime}, \eta\right\}\right) \sigma^{2}$, then change of the population growth rate will not change the properties of the long-run economic growth and the phenomenon that the long-run growth rate will converge to zero. Therefore, only the population growth can not determine the long-run economic trend. When the noise of the population growth is sufficiently small or its expectation is sufficiently high, it is possible for the economy to show the positive long-run economic growth.

Theorem 3 show that the system (SP) will converge to origin point if the expectation of the population growth rate is sufficiently low or its uncertainty is sufficiently large. But, in comparison with its uncertainty, if the expected population growth rate is 
sufficiently high, whether it is possible for both the technological progress and the capital accumulation to show the positive long-run economic growth or not? The following estimation and the next theorem may partly answer the question.

Theorem 4 When $\theta+\xi<0$ is retained,

$$
\limsup _{t \rightarrow+\infty} \frac{\log \left(x(t)-\frac{2 \theta+\xi}{\alpha^{\prime}} y(t)\right)}{\log t} \leq 1
$$

further, if $2 n>\sigma^{2}$,

$$
\liminf _{t \rightarrow+\infty} \frac{\log \left(x(t)-\frac{2 \theta+\xi}{\alpha^{\prime}} y(t)\right)}{\log t} \geq-\frac{\max \left\{\alpha^{\prime}, \eta\right\} \sigma^{2}}{2 n-\sigma^{2}} \vee\left(-\frac{1}{2}\right) .
$$

The proof of (6) and (7) may be found in [14]. We make a small progress and obtain a more precious estimation on the proof of (7). For the convenience of reference, we put their proofs into appendix A.

(6) and (7) mean that for any $\varepsilon>0$, there exists a positive random variable $T_{\varepsilon}$ such that, with probability one,

$$
t^{-\delta-\varepsilon} \leq x(t)-\frac{2 \theta+\xi}{\alpha^{\prime}} y(t) \leq t^{1+\varepsilon}, \quad \forall t>T_{\varepsilon},
$$

where $\delta=\left[\max \left\{\alpha^{\prime}, \eta\right\} \sigma^{2} /\left(2 n-\sigma^{2}\right)\right] \wedge 1 / 2$. The right inequality means, with probability one, the economic growth rate will not fluctuate faster than $t^{1+\varepsilon}$, and at most, at polynomial growth speed. This means that the long-run growth rates of the technological progress and capital accumulation will not change very fast. We know, if the expected population growth rate, in comparison with its uncertainty, is sufficiently low, the system (SP) will converge to zero. The left inequality tells us that, if the expected population growth rate is sufficiently high or its uncertainty is sufficiently small, the long-run economic growth rate will not decay very fast, at most, with the polynomial speed. Therefore, if the uncertainty of the population growth rate, in comparison with its expectation, is sufficiently small, i.e., the population growth is relatively stationary, the fluctuation of the economic growth will not be very violent.

According to these results above, the paper may obtain the explicit sample average of the growth rates of both the technological progress and capital accumulation in time, which is the most important result in the paper and may be expressed as

Theorem 5 When $\theta+\xi<0$ is retained and $2 n>\sigma^{2}$, the sample average of the growth rates of both the technological progress and the capital accumulation in time are 
respectively

$$
\begin{aligned}
& \bar{x}=\lim _{t \rightarrow+\infty} \frac{1}{t} \int_{0}^{t} x(s) d s=-\frac{\eta+\xi}{2(\theta+\xi)}\left(2 n-\sigma_{L}^{2}\right), \\
& \bar{y}=\lim _{t \rightarrow+\infty} \frac{1}{t} \int_{0}^{t} y(s) d s=-\frac{\eta-\theta}{2(\theta+\xi)}\left(2 n-\sigma_{L}^{2}\right) .
\end{aligned}
$$

Proof Define $Z(t)=(\log x(t), \log y(t))^{T}$, and apply Itô formula to $Z(t)$, then

$$
d Z(t)=\left[b-\frac{\sigma^{2}}{2}\left(\eta^{2}, \alpha^{2}\right)^{T}+A X(t)\right] d t+\beta d w(t)
$$

substituting the expression of $b$ into this equation yields

$$
\frac{Z(t)-Z(0)}{t}=\frac{1}{2}\left(2 n-\sigma^{2}\right)\left(\eta, \alpha^{\prime}\right)^{T}+A \frac{1}{t} \int_{0}^{t} X(s) d s+\frac{\beta w(t)}{t} .
$$

From Theorem 4,

$$
\lim _{t \rightarrow \infty} \frac{\log x(t)}{t}=\lim _{t \rightarrow \infty} \frac{\log y(t)}{t}=0, \text { a.s. }
$$

Noting that $\lim _{t \rightarrow \infty} w(t) / t=0$ from law of the iterated logarithm (see [12] P16), so

$$
\lim _{t \rightarrow} \frac{1}{t} \int_{0}^{t} X(s) d s=-\frac{1}{2}\left(2 n-\sigma^{2}\right) A^{-1}\left(\eta, \alpha^{\prime}\right)^{T}=-\frac{2 n-\sigma^{2}}{2(\theta+\xi)}(\eta+\xi, \eta-\theta)^{T},
$$

which is the desired assertions.

The results shows, although the road to prosperity is not a smooth one, if uncertainty of the population growth, in comparison with its expected growth rate, is sufficiently small, it still shows an upward trend average growth in the long term. The sample average of the growth rates completely depends on the difference between the expected population growth rate and its uncertainty. The more difference means the higher sample average of the growth rates.

In classical Solow [20] and Ramsey [16] models, the growth rate of aggregate capital accumulation is completely determined by the exogenous population growth and technological progress. When technological progress is endogenized as that in the section two of this paper, if $\theta+\xi<0$, the growth rates of both the capital accumulation and the technological progress are completely determined by the population growth rate. From Theorem 5, in the stochastic R\&D model, the crucial factor of the sample average of the economic growth rate is the difference between the population growth rate and its uncertainty, not only the population rate. Therefore, when we consider the economic growth, the uncertainty should be an important factor. 
Comparing the explicit expressions of the sample average of the growth rates of both the technological progress and capital accumulation with their deterministic counterparts $\left(x^{*}, y^{*}\right)$, it is easy to see

$$
\bar{x}=x^{*}+\frac{\eta+\xi}{2(\theta+\xi)} \sigma^{2}, \quad \bar{x}=y^{*}+\frac{\eta-\theta}{2(\theta+\xi)} \sigma^{2},
$$

that is to say, in the long term, the sample average of the growth rates of both the technological progress and capital accumulation may be expressed as the difference between the steady-state value in the corresponding deterministic model and a product of a positive number and the uncertainty, so the uncertainty decreases the sample average of the economic growth rate. When uncertainty of population growth is zero, these sample average of the stochastic growth rates returns to their deterministic counterparts. From the expression of $\bar{x}, \bar{y}$, all factors that increases or decrease the steady-state economic growth rate in the deterministic $R \& D$ model still have the same effect on the sample average of the stochastic economic growth rate. Therefore, it is very important to do one's best for governments to eliminate uncertainty and retain the stationary economic development.

Different from other papers (for example, [15], [3]) in which they compare the expectation of the variables in the stochastic models with their counterparts in the corresponding deterministic model when they examine the relation between the deterministic and corresponding stochastic models, we compare the sample average value in time with its deterministically steady-state counterparts. When we discuss the economic growth of a country, it is more easy to obtain the sample data, so maybe it is more convenient to compare the theoretical values with these real values.

Canton [6] believes that, in an uncertain economic environment, the economic system will show higher growth rate, since people devote more time to learning activities and save more money for future uncertainty. In the Merton's [15] model, there are the higher capital-labor ratio, per capital output and capital-output and the less interest rate than their counterparts in the corresponding deterministic model. In sum, in these models, uncertainty is a positive factor in the economic system. By introduction of uncertainty from the creation of knowledge, Hek [9] obtain a negative link between the uncertainty and the economic growth. In the paper, we may obtain the negative link between the uncertainty and the sample average of the economic growth rate when the uncertainty comes from the population growth and explicitly compute the relation between the average sample average of the growth rate of the system and the steady-state growth rate in the corresponding deterministic model. 


\section{Concluding remarks}

Using the recent mathematical technique for the stochastic differential equations, the paper discusses the stochastic R\&D model that may express as a stochastic LotkaVolterra system. The paper obtains a condition that may ensure that there exists a globally positively steady state for the corresponding deterministic system, may also ensure that the stochastic Lotka-Volterra system exists a uniquely globally positive solution. The result means, when the deterministic R\&D model is extended into the stochastic version, the economic system still shows the positive growth. At the same time, the paper finds the economic growth will not fluctuate very fast, at most, with the polynomial speed in the long run.

The population growth always plays an important role during the economic growth process. In the paper, the growth rates of both the technological progress and the capital accumulation in the long run is controlled by the difference between the expected population growth rate and its uncertainty. The less difference means the less growth rates of both the technological progress and capital accumulation. Furthermore, when the expected population growth rate is sufficiently low or the uncertainty is sufficiently large, the stochastic economic growth rate will converge to zero, which means that the growth rates of both the technological progress and the capital accumulation will be zero in the long run.

When the population growth is relatively stationary, that is to say, if the uncertainty is sufficiently low or its expectation is sufficiently high, the economic growth will not decay very fast, at most with the polynomial speed. Based on the result, we explicitly compute the sample average of the growth rates of both the technological progress and capital accumulation which may express as the difference between the steady-state value in the corresponding deterministic model and a product of a positive number and the uncertainty such that we may clearly obtain that the higher expected population growth rate and the less uncertainty mean the higher sample average of the growth

rates. To our knowledge, there is no paper to use this way to consider economic growth since most papers examine the relation between the stochastic models and the corresponding deterministic models by the expectation, not the sample average value.

The paper only discusses the uncertainty from the population growth. If there exist more origins of the uncertainty, maybe there exists more complicated relation between the growth and uncertainty, which will be our next work. 


\section{Appendix A}

Proof of (6) According to the Eq. (4) and $\theta+\xi<0$,

$$
d V(x(t), y(t)) \leq \max \left\{b_{x}, b_{y}\right\} V(x(t), y(t)) d t+\sigma[\eta x(t)-(2 \theta+\xi) y(t)] d w(t),
$$

SO

$$
\begin{aligned}
E\left(\sup _{t \leq u \leq t+1} V(x(u), y(u))\right) \leq & E V(x(t), y(t))+\max \left\{\left|b_{x}\right|,\left|b_{y}\right|\right\} \int_{t}^{t+1} E V(x(s), y(s)) d s \\
& +E\left(\sup _{t \leq u \leq t+1} \int_{t}^{u} \sigma[\eta x(s)-(2 \theta+\xi) y(s)] d w(s)\right) .
\end{aligned}
$$

By the well-known Burkholder-Davis-Gundy inequality, we derive that

$$
\begin{aligned}
& E\left(\sup _{t \leq u \leq t+1} \int_{t}^{u} \sigma[\eta x(s)-(2 \theta+\xi) y(s)] d w(s)\right) \\
\leq & 6 \sigma E\left(\int_{t}^{t+1}[\eta x(s)-(2 \theta+\xi) y(s)]^{2} d s\right)^{\frac{1}{2}} \\
\leq & 6 \sigma \max \left\{\alpha^{\prime}, \eta\right\} E\left(\int_{t}^{t+1} V^{2}(x(s), y(s)) d s\right)^{\frac{1}{2}} \\
\leq & 6 \sigma \max \left\{\alpha^{\prime}, \eta\right\} E\left(\sup _{t \leq u \leq t+1} V(x(u), y(u)) \int_{t}^{t+1} V(x(s), y(s)) d s\right)^{\frac{1}{2}} \\
\leq & \frac{1}{2} E\left(\sup _{t \leq u \leq t+1} V(x(u), y(u))\right)+18 \sigma^{2} \max \left\{\alpha^{\prime 2}, \eta^{2}\right\} \int_{t}^{t+1} E V(x(s), y(s)) d s .
\end{aligned}
$$

From Theorem 2, there exists a positive constant $L$ such that $\lim _{\sup _{t \rightarrow \infty}} E V(x(t), y(t)) \leq$ $L$, so

$$
E\left(\sup _{t \leq u \leq t+1} V(x(u), y(u))\right) \leq 2\left(1+\max \left\{\left|b_{x}\right|,\left|b_{y}\right|\right\}+18 \sigma^{2} \max \left\{\alpha^{\prime 2}, \eta^{2}\right\}\right) L .
$$

Therefore, for any $\varepsilon>0$, by the well-known Chebyshev inequlity, we have

$P\left\{\left(\sup _{k \leq t \leq k+1} V(x(t), y(t))\right)>k^{1+\varepsilon}\right\} \leq \frac{2\left(1+\max \left\{\left|b_{x}\right|,\left|b_{y}\right|\right\}+18 \sigma^{2} \max \left\{\alpha^{\prime 2}, \eta^{2}\right\}\right) L}{k^{1+\varepsilon}}, k=1,2, \cdots$.

Applying the well-known Borel-Cantelli Lemma (see [12], P7), we obtain that for almost all $\omega \in \Omega$,

$$
\sup _{k \leq t \leq k+1} V(x(t), y(t)) \leq k^{1+\varepsilon}
$$

holds for all but finitely many $k$. Hence, there exists a $k_{0}(\omega)$, for almost all $\omega \in \Omega$, (13) holds for whenever $k \geq k_{0}$. Consequently, for almost all $\omega \in \Omega$, if $k \geq k_{0}$ and $k \leq t \leq k+1$,

$$
\frac{\log \left[x(t)-\frac{2 \theta+\xi}{\alpha^{\prime}} y(t)\right]}{\log y(t)} \leq \frac{(1+\varepsilon) \log k}{\log t} \leq \frac{(1+\varepsilon) \log k}{\log k}=1+\varepsilon .
$$


Letting $\varepsilon \rightarrow 0$, we obtain the desire result. The proof is therefore complete.

Proof of (7) We still choose the same function $V(x(t), y(t))$ as before, then

$$
\begin{aligned}
d\left(\frac{1}{V(x(t), y(t))}\right) \leq & -\frac{1}{V^{2}(x(t), y(t))}\left[\left(\theta x^{2}(t)-2 \theta x(t) y(t)+(2 \theta+\xi) y^{2}(t)+b_{x} x(t)\right.\right. \\
& \left.\left.-\frac{2 \theta+\xi}{\alpha^{\prime}} b_{y} y(t)\right) d t+\sigma[\eta x(t)-(2 \theta+\xi) y(t)] d w(t)\right] \\
& +\frac{\sigma^{2}}{V^{3}(x(t), y(t))}[\eta x(t)-(2 \theta+\xi) y(t)]^{2} d t .
\end{aligned}
$$

Applying the Itô formula again, for any $\varepsilon>0$, we have

$$
\begin{aligned}
& d\left(1+\frac{1}{V(x(t), y(t))}\right)^{\varepsilon} \\
= & \varepsilon\left(1+\frac{1}{V(x(t), y(t))}\right)^{\varepsilon-2}\left\{\left(1+\frac{1}{V(x(t), y(t))}\right) d\left(\frac{1}{V(x(t) y(t))}\right)-\frac{1-\varepsilon}{2}\left[d\left(\frac{1}{V(x(t), y(t))}\right)\right]^{2}\right\} \\
= & \varepsilon\left(1+\frac{1}{V(x(t), y(t))}\right)^{\varepsilon-2}\left\{-\left(\frac{1}{V^{2}(x(t), y(t))}+\frac{1}{V^{3}(x(t), y(t))}\right)\left[\left(\theta x^{2}(t)-2 \theta x(t) y(t)\right.\right.\right. \\
& \left.\left.+(2 \theta+\xi) y^{2}(t)+b_{x} x(t)-\frac{2 \theta+\xi}{\alpha^{\prime}} b_{y} y(t)\right)\right] \\
& \left.+\left(\frac{\sigma^{2}}{V^{3}(x(t), y(t))}+\frac{1+\varepsilon}{2} \frac{\sigma^{2}}{V^{4}(x(t), y(t))}\right)[\eta x(t)-(2 \theta+\xi) y(t)]^{2}\right\} d t \\
& -\varepsilon\left(1+\frac{1}{V(x(t), y(t))}\right)^{\varepsilon-1} \frac{\sigma}{V^{2}(x(t), y(t))}[\eta x(t)-(2 \theta+\xi) y(t)] d w(t)
\end{aligned}
$$

It is easy to see that for all $x, y \in \mathbf{R}_{+}$, there exists a positive constant $K_{1}$,

$$
\begin{aligned}
& -\frac{\theta x^{2}-2 \theta x y+(2 \theta+\xi) y^{2}}{V^{2}(x, y)} \leq K_{1} ; \\
& \frac{\sigma^{2}(1+\varepsilon)[\eta x-(2 \theta+\xi) y]^{2}}{2 V^{2}(x, y)}-\frac{b_{x} x-\frac{2 \theta+\xi}{\alpha^{\prime}} b_{y} y}{V(x, y)} \leq \max \left\{\frac{1+\varepsilon}{2} \eta^{2} \sigma^{2}-b_{x}, \frac{1+\varepsilon}{2} \alpha^{\prime 2} \sigma^{2}-b_{y}\right\},
\end{aligned}
$$

Substituting these inequalities into Eq. (10) yields

$$
d\left(1+\frac{1}{V(x(t), y(t))}\right)^{\varepsilon} \leq \varepsilon\left(1+\frac{1}{V(x(t), y(t))}\right)^{\varepsilon-2} H(x(t), y(t)) d t-M(t) d w(t),
$$

where

$$
\begin{aligned}
H(x(t), y(t))= & \max \left\{\frac{1+\varepsilon}{2} \eta^{2} \sigma^{2}-b_{x}, \frac{1+\varepsilon}{2} \alpha^{\prime 2} \sigma^{2}-b_{y}\right\} \frac{1}{V^{2}(x(t), y(t))} \\
& +\left(K_{1}+\max \left\{\eta^{2} \sigma^{2}-b_{x}, \alpha^{\prime 2} \sigma^{2}-b_{y}\right\}\right) \frac{1}{V(x(t), y(t))}+K_{1},
\end{aligned}
$$




$$
M(t)=\frac{\varepsilon \sigma}{V^{2}(x(t), y(t))}\left(1+\frac{1}{V(x(t), y(t))}\right)^{\varepsilon-1}[\eta x(t)-(2 \theta+\xi) y(t)]
$$

Choose

$$
0<\varepsilon<\frac{2 n-\sigma^{2}}{\sigma^{2} \max \left\{\alpha^{\prime}, \eta\right\}} \wedge 2
$$

for $\max \left\{(1+\varepsilon) \eta^{2} \sigma^{2} / 2-b_{x},(1+\varepsilon) \alpha^{2} \sigma^{2} / 2-b_{y}\right\}<0$, then there exists a positive constant $K_{2}$ such that

$$
\varepsilon\left(1+\frac{1}{V(x(t), y(t))}\right)^{\varepsilon-2} H(x(t), y(t))<K_{2},
$$

So,

$$
d\left(1+\frac{1}{V(x(t), y(t))}\right)^{\varepsilon} \leq K_{2} d t-M(t) d w(t) .
$$

Then, by the Itô formula,

$$
\begin{aligned}
& d\left[e^{\gamma t}\left(1+\frac{1}{V(x(t), y(t))}\right)^{\varepsilon}\right] \\
= & e^{\gamma t}\left[\gamma\left(1+\frac{1}{V(x(t), y(t))}\right)^{\varepsilon} d t+d\left(1+\frac{1}{V(x(t), y(t))}\right)^{\varepsilon}\right] \\
= & e^{\gamma t}\left(1+\frac{1}{V(x(t), y(t))}\right)^{\varepsilon-2}\left[\gamma\left(1+\frac{1}{V(x(t), y(t))}\right)^{2}+\varepsilon H(x(t), y(t))\right] d t-e^{\gamma t} M(t) d w(t) .
\end{aligned}
$$

Choose

$$
0<\gamma<-\varepsilon \max \left\{\frac{1+\varepsilon}{2} \eta^{2} \sigma^{2}-b_{x}, \frac{1+\varepsilon}{2} \alpha^{\prime 2} \sigma^{2}-b_{y}\right\},
$$

then there exists a positive constant $K$ such that

$$
\left(1+\frac{1}{V(x(t), y(t))}\right)^{\varepsilon-2}\left[\gamma\left(1+\frac{1}{V(x(t), y(t))}\right)^{2}+\varepsilon H(x(t), y(t))\right] \leq K,
$$

So

$$
E\left[e^{\gamma t}\left(1+\frac{1}{V(x(t), y(t))}\right)^{\varepsilon}\right] \leq E\left(1+\frac{1}{V(x(0), y(0))}\right)^{\varepsilon}+\frac{K}{\gamma}\left(e^{\gamma t}-1\right),
$$

which implies that

$$
E\left(1+\frac{1}{V(x(t), y(t))}\right)^{\varepsilon} \leq \frac{K}{\gamma}
$$

Eq. (11) implies

$$
E\left(\sup _{t \leq u \leq t+1}\left(1+\frac{1}{V(x(u), y(u))}\right)^{\varepsilon}\right) \leq E\left(1+\frac{1}{V(x(t), y(t))}\right)^{\varepsilon}+K_{2}+E\left(\sup _{t \leq u \leq t+1}\left|\int_{t}^{u} M(s) d w(s)\right|\right) .
$$


By the well-known Burkholder-Davis-Gundy inequality,

$$
\begin{aligned}
& E\left(\sup _{t \leq u \leq t+1}\left|\int_{t}^{u} M(s) d w(s)\right|\right) \\
\leq & 6 \varepsilon \sigma E\left(\int_{t}^{t+1}\left(1+\frac{1}{V(x(s), y(s))}\right)^{2 \varepsilon-2} \frac{1}{V^{4}(x(s), y(s))}[\eta x(t)-(2 \theta+\xi) y(t)]^{2} d s\right)^{\frac{1}{2}} \\
\leq & 6 \varepsilon \sigma \max \left\{\alpha^{\prime}, \eta\right\} E\left[\int_{t}^{t+1}\left(1+\frac{1}{V(x(s), y(s))}\right)^{2 \varepsilon} d s\right]^{\frac{1}{2}} \\
\leq & 6 \varepsilon \sigma \max \left\{\alpha^{\prime}, \eta\right\} E\left[\sup _{t \leq u \leq t+1}\left(1+\frac{1}{V(x(u), y(u))}\right)^{\varepsilon} \int_{t}^{t+1}\left(1+\frac{1}{V(x(s), y(s))}\right)^{\varepsilon} d s\right]^{\frac{1}{2}} \\
\leq & \frac{1}{2} E\left(\sup _{t \leq u \leq t+1}\left(1+\frac{1}{V(x(u), y(u))}\right)^{\varepsilon}\right)+18 \varepsilon^{2} \sigma^{2} \max \left\{\alpha^{\prime 2}, \eta^{2}\right\} \int_{t}^{t+1} E\left(1+\frac{1}{V(x(s), y(s))}\right)^{\varepsilon} d s .
\end{aligned}
$$

Therefore,

$$
E\left(\sup _{t \leq u \leq t+1}\left(1+\frac{1}{V(x(u), y(u))}\right)^{\varepsilon}\right) \leq 2\left[\frac{K}{\gamma}\left(1+18 \varepsilon^{2} \sigma^{2} \max \left\{\alpha^{\prime 2}, \eta^{2}\right\}\right)+K_{2}\right]<+\infty .
$$

Using the same way as the proof of (6),

$$
\limsup _{t \rightarrow+\infty} \frac{\log \left(1+\frac{1}{V(x(t), y(t))}\right)^{\varepsilon}}{\log t} \leq 1, \text { a.s. }
$$

Hence, this implies

$$
\limsup _{t \rightarrow+\infty} \frac{\log V^{-\varepsilon}(x(t), y(t))}{\log t} \leq \limsup _{t \rightarrow+\infty} \frac{\log \left(1+\frac{1}{V(x(t), y(t))}\right)^{\varepsilon}}{\log t} \leq 1, \quad \text { a.s. }
$$

which further implies

$$
\liminf _{t \rightarrow+\infty} \frac{\log V(x(t), y(t))}{\log t} \geq-\frac{1}{\varepsilon}, \text { a.s. }
$$

Because this holds for any $\varepsilon$ that obeys its interval, we must therefore have the Eq. (14). The proof is complete.

\section{References}

[1] P. Aghion, P. Howitt, A model of growth through creative destruction, Econometrica 60 (1992) $323-351$.

[2] A. Bahar, X. Mao, Stochastic delay population dynamics, International Journal of Pure and Applied Mathematics, 4 (2004) 377-400.

[3] M. Binder, M. H. Pesaran, Stochastic growth models and their econometric implications, Journal of Economic Growth, 4 (1999), 139-183. 
[4] F. Bourguignon, A particular calss of continuous-times tochastic growth models, Journal Economic Theory, 9 (1974) 141-158.

[5] A. Berman and R. J. Plemmons, Nonnegative matrices in the mathematicial sciences, SIAM, 1994.

[6] E. Canton, Business cycles in a two-sector model of endogenous growth, Economic Theory, 19 (2002), 477-492.

[7] K. B. Grier, G. Tullock, An empirical analysis of cross-national economic growth, 1951-1980, Journal of Monetary economics, 24 (1989), 259-276.

[8] G. M. Grossman, E. Helpman, Innovation and growth in the global economy, Cambridge: MIT Press, 1991.

[9] P. A. de Hek, On endogenous growth under uncertainty, International Economic Review, 40 (1999) 727-744.

[10] C. L. Jones, R\&D-based models of economic growth, Journal of Political Economiy, 103 (1995) 759-784.

[11] R. L. Kormendi, P. G. Meguire, Macroeconomic determinants of grwoth: cross-country evidence, Journal of Monetary Economics, 16 (1985), 141-163.

[12] X. Mao, Stochatic differential equations and applications, Horwood, 1997.

[13] X. Mao, G. Marion, E. Renshaw, Environmental Brownian noise suppresses explosions in populations dynamics, Stochastic Process and Application. 97 (2002) 95-110.

[14] S. Pang, F. Deng and X. Mao, Asymptotic properties of stochastic population dynamics, 2006, forthcoming.

[15] R. Merton, An asymptotic theory of growth under uncertainty, Review Economic Studies, 42 (1975) 375-393.

[16] F. P. Ramsey, A mathematical theory of saving, Economic Journal, 38 (1928) 534-559.

[17] G. Ramsey, V. A. Ramsey, Cross-country evidence on the link between volatility and growth, American Economic Review 85 (1995), 1138-1151.

[18] D. Romer, Advanced macroeconoomics, the McGraw-Hill Companies, Inc. 1996.

[19] P.M. Romer, Endogenous Technological Change, Journal of Political Economy, 98 (1990) S71S102.

[20] R. M. Solow, A contribution to the theory of economic growth, Quarterly Journal of Economics 70 (1956) 65-94. 
[21] V. Zarnowitz, G. Moore, Major changes in cyclical Behavior, the American Business Cycle: continuity and change. (Chicago: University of Chicago Press, 1986), pp. 519-572. 